ARTIGO ORIGINAL ORIGINAL ARTICLE

\title{
Análise de custo-efetividade de pirfenidona em comparação a nintedanibe no tratamento de fibrose pulmonar idiopática na perspectiva do sistema suplementar de saúde brasileiro
}

\author{
Cost-effectiveness analysis of pirfenidone versus nintedanib \\ on the treatment of idiopathic pulmonary fibrosis in the \\ perspective of the Brazilian private healthcare system \\ Rodrigo Shimabukuro Ho', Camila Rufino Souza', Cintia S. Kurokawa La Scala \\ de Oliveira', Claudio Maurício Lisondo', Marcia Regina Dias Alves \\ DOI: 10.21115/JBES.v9.suppl1.89-95
}

\section{Palavras-chave:}

pirfenidona, fibrose pulmonar idiopática, nintedanibe, custo-efetividade

\section{Keywords:}

pirfenidone, idiopathic pulmonar fibrosis, nintedanib, cost-effectiveness

\begin{abstract}
RESUMO
Objetivo: $O$ objetivo do estudo foi avaliar o custo-efetividade de pirfenidona em comparação ao nintedanibe no tratamento de pacientes com fibrose pulmonar idiopática (FPI) na perspectiva do sistema suplementar de saúde. Métodos: $\bigcirc$ modelo foi realizado considerando um horizonte de tempo lifetime. O principal desfecho da análise foram os anos de vida ganhos. Os custos de aquisição de medicamentos foram obtidos por meio das listas oficiais de preço, considerando o ICMS 18\%. Os demais custos relacionados ao manejo da doença, transplante de pulmão e custo de final de vida foram calculados por um microcusteio baseado em opinião de especialistas e listas de preços de procedimentos. Os dados de eficácia foram extraídos dos estudos CAPACITY 1, 2 e ASCEND, e extrapolados por meio de uma distribuição paramétrica Weibull. Os dados referentes ao nintedanibe foram extrapolados por uma comparação indireta. Resultados: Os custos totais de pirfenidona e de nintedanibe foram $\mathrm{R} \$ 319.689$ e $\mathrm{R} \$ 522.887$, respectivamente. Os anos de vida salvos resultantes foram 6,536 para pirfenidona e 5,726 para nintedanibe, resultando em um valor incremental de 0,810. Conclusão: Dessa maneira, a partir dos valores incrementais de custos e efetividade, a pirfenidona demonstrou ser uma opção terapêutica dominante quando comparada ao nintedanibe.
\end{abstract}

\begin{abstract}
Objective: The objective of the study was to evaluate the cost-effectiveness of pirfenidone in comparison to nintedanib in the treatment of patients with idiopathic pulmonary fibrosis (IPF) under the Brazilian private healthcare system perspective. Methods: The model was performed considering a lifetime time horizon. The main outcome was life years (LY) gained. The drug acquisition costs were obtained from official price lists, considering taxes of $18 \%$. The other related costs (disease management, lung transplantation and end-of-life costs) were calculated by a micro-costing based on specialists' opinion and procedures price lists, costs are presented in 2017 (R\$). The efficacy data was extracted from CAPACITY 1,2 and ASCEND studies, and extrapolated by the parametric distribution Weibull. The data related to nintedanib was extrapolated by an indirect comparison. Results: The total costs of pirfenidone and nintedanibe were $R \$ 319,689$ and $R \$ 522,887$, respectively. The LY results were 6.536 for pirfenidone and 5.726 for nintedanib, resulting in an incremental value of 0.810. Conclusion: Therefore, pirfenidone demonstrated to be a dominant therapeutic option when compared to nintedanib, based on incremental values of cost and effectiveness.
\end{abstract}

Recebido em: 07/07/2017. Aprovado para publicação em: 16/08/2017

1. F. Hoffmann-La Roche Ltd., São Paulo, SP, Brasil.

Instituição onde o trabalho foi executado: F. Hoffmann-La Roche Ltd. e SENSE Company.

Financiamento: $O$ estudo foi financiado pela F. Hoffmann-La Roche Ltd.

Autor correspondente: Marcia Regina Dias Alves. Av. Engenheiro Billings, 1729, Jaguaré - São Paulo, SP, Brasil. CEP: $05321-900$.

E-mail: marcia.alves@roche.com 


\section{Introdução}

A fibrose pulmonar idiopática (FPI) é uma doença rara, crônica e irreversível, caracterizada por deposição de tecido cicatricial nos pulmões e dispneia progressiva. (Frankel \& Schwarz, 2009; Nalysnyk et al., 2012; Raghu et al., 2015). Em todo o mundo, aproximadamente 5 milhões de pessoas são acometidas com FPI, com prevalência mais elevada entre homens do que entre mulheres (20 por 100.000 e 13 por 100.000, respectivamente) (Meltzer \& Noble, 2008), com diagnóstico, em média, aos 66 anos (American Thoracic Society (ATS) \& European Respiratory Society (ERS), 2000; du Bois, 2012).

Os sintomas clínicos da FPI são inespecíficos e podem ser confundidos com outras doenças pulmonares e cardíacas. A manifestação clínica inicial ocorre de maneira insidiosa com piora progressiva, e os sintomas mais comuns são a dispneia progressiva e a tosse crônica, geralmente seca (American Thoracic Society (ATS) \& European Respiratory Society (ERS), 2000). Aproximadamente 5\% dos pacientes não apresentam sintomas, e a doença é diagnosticada por acaso (Medscape, 2016).

A progressão da FPI de doença assintomática para sintomática pode levar muitos anos (Adkins \& Collard, 2012; Raghu et al., 2015). Entretanto, o curso da FPI, na maioria das vezes, é inexorável rumo ao óbito por insuficiência respiratória com hipoxemia grave ou devido a outras enfermidades relacionadas com as complicações da própria fibrose pulmonar. A sobrevida média após o surgimento dos primeiros sintomas é cerca de dois a cinco anos, e, após o diagnóstico estabelecido, a sobrevida média é de 30 meses (Bjoraker et al., 1998; Ley et al., 2011; Meltzer \& Noble, 2008; Nunes et al., 2011). No entanto, é difícil predizer o curso da doença em cada paciente. Cerca de 20 a 25\% dos pacientes podem apresentar um curso prolongado da doença e com sobrevida de cinco anos ou mais, no entanto, é difícil predizer quem evoluirá desse modo, pois a caracterização desse fenótipo de doença só é possível de ser reconhecida retrospectivamente.

A taxa de mortalidade global devido à FPI aumentou ao longo dos anos, e a maioria das mortes ocorre por progressão da fibrose pulmonar e não necessariamente por comorbidades que comumente ocorrem (Lee et al., 2014; Ley et al., 2011).

A insuficiência respiratória é a causa mais comum de morte (aproximadamente 60\%), mais comumente devido à exacerbação aguda da FPI (29\%), pneumonia (15\%) e FPI cronicamente progressiva (12\%) (Lee et al., 2014).

Como opções terapêuticas, o transplante pulmonar e a suplementação de oxigênio apresentam recomendação positiva para o tratamento de FPI, entretanto, também apresentam diversas desvantagens e restrições (Raghu et al., 2011, 2015). No caso do transplante pulmonar, o procedimento pode ser realizado em apenas uma parcela de pacientes devido à restrição de possíveis comorbidades associadas à idade avançada dos pacientes com FPI, além dos riscos ine- rentes à cirurgia e os custos elevados (Adkins \& Collard, 2012; Kistler et al., 2014; Nalysnyk et al., 2012a).

A terapia com suplementação de oxigênio, apesar de promover alguns benefícios, apresenta alguns inconvenientes para os pacientes, e os custos envolvidos ainda são imprecisos (Raghu et al., 2015). Em termos econômicos, a FPI, mesmo sendo uma doença rara, tem elevado impacto econômico (Mooney et al., 2016; Visca et al., 2011).

Segundo Mooney et al., 2016 (Mooney et al., 2016), os pacientes hospitalizados com diagnóstico de FPI permaneceram mais de uma semana no hospital em comparação com pacientes hospitalizados com outros diagnósticos de admissão, com um custo médio de US\$20.000 e mais de 10\% dos pacientes que morreram antes da alta hospitalar.

Assim, a FPI constitui uma doença crônica, progressiva, com um curso inexorável e devastador, que apresenta um desfecho fatal, com impacto negativo na qualidade de vida dos pacientes e de suas famílias e elevada carga econômica imposta ao sistema de saúde (Frankel \& Schwarz, 2009; Nalysnyk et al., 2012; Raghu et al., 2015).

Como tratamento farmacológico para FPI, de acordo com a última atualização (2015) da diretriz das sociedades Americana (ATS), Europeia (ERS), Japonesa (JRS) e Latino-americana (ALAT), é recomendado o uso de pirfenidona ou de nintedanibe como opções terapêuticas para a FPI (Raghu et al., 2015).

A pirfenidona e nintedanibe foram as únicas opções que demonstraram eficácia em ensaios clínicos randomizados (Richeldi et al., 2011; Stowasser et al., 2015). Até o momento, não existem estudos head-to-head que comparem esses medicamentos diretamente. Ambas as medicações apresentam eficácia semelhante em termos de reduzir o declínio da função pulmonar em pacientes com FPI. No entanto, os dados agrupados publicados sobre o nintedanibe não mostraram nenhum efeito significativo sobre a mortalidade, demonstraram somente uma redução no declínio da capacidade vital forçada (CVF) (Richeldi et al., 2011). Diferentemente dos dados de estudos clínicos disponíveis para pirfenidona, que demostraram tanto uma redução da mortalidade quanto uma meIhora em relação às taxas de declínio da CVF (Stowasser et al., 2015).

O objetivo desse estudo foi avaliar o custo-efetividade de pirfenidona em comparação ao nintedanibe no tratamento de pacientes com FPI na perspectiva do sistema suplementar de saúde brasileiro.

\section{Métodos}

Um modelo de Markov foi elaborado para estimativa de custos e desfechos dos tratamentos comparados. O modelo acompanhou os pacientes com FPI, iniciando o tratamento ao longo do curso natural da doença até o final de suas vidas. As transições dos pacientes em diferentes estados estão 
representadas na Figura 1. O horizonte de tempo considerado na análise foi lifetime, sendo considerado um tempo de acompanhamento de 33 anos, com idade inicial média do paciente de 67 anos. O ciclo do modelo foi de três meses.

Os pacientes podiam permanecer em estado livre de progressão - definida como aqueles pacientes que não tiveram declínio confirmado de $10 \%$ ou mais da CVF predita em \% (CVF\% pred), redução maior ou igual a 50 metros no teste de esforço - ou apresentar progressão da doença, com a ocorrência de um dos eventos citados anteriormente, com exceção da morte. Em estado de progressão, os pacientes podiam passar para o estado de transplante de pulmão. Além disso, todos os pacientes podiam progredir para a morte a partir de qualquer estado do modelo.

Uma taxa de desconto anual de 5\% para custos e para desfechos foi aplicada, de acordo com as recomendações das Diretrizes Metodológicas para Estudos de Avaliação Econômica de Tecnologias em Saúde, publicadas pelo Ministério da Saúde (Ministério da Saúde, Brasil. Secretária de Ciência, Tecnologia e Insumos Estratégicos. Departamento de Ciência e Tecnologia, 2014).

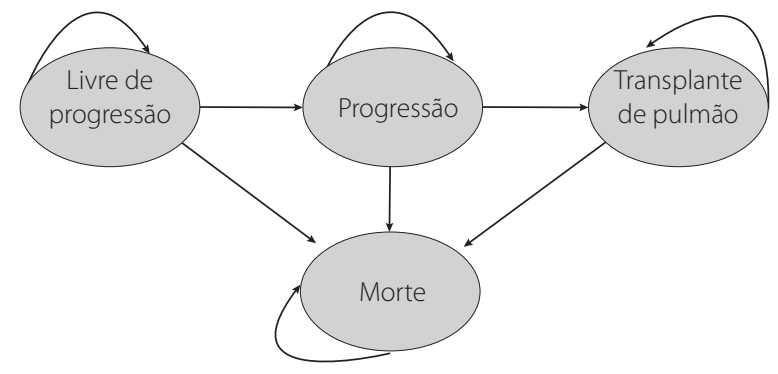

Figura 1. Estrutura do modelo de Markov.

\section{Dados clínicos}

Na ausência de dados de comparação direta (head-to-head) para avaliação de pirferidona e de nintedanibe, uma comparação indireta realizada por revisão sistemática e metanálise em rede Bayesiana (NMA, do inglês network meta-analysis) foi utilizada para os dados clínicos de eficácia (Fleetwood et al., 2017). A revisão sistemática foi conduzida até abril de 2015 e os ensaios clínicos elegíveis foram os randomizados de fase II/III para pacientes adultos com FPI. Nesta NMA, dez estudos foram analisados, sendo que cinco destes ensaios avaliaram pirfenidona versus placebo (CAPATICTY 1, CAPACITY 2, SP2, SP3 e ASCEND), e três compararam nintedanibe versus placebo (TOMORROW, INPULSIS 1 e INPULSIS 2).

Para alteração na CVF a partir da linha de base, a NMA indicou que pirfenidona e nintedanibe foram mais eficazes do que placebo em 1 ano (pirfenidona versus placebo: diferença $=0,12$ litro, intervalo de confiança de 95\%: 0,03 a 0,21 L; nintedanibe versus placebo: diferença $=0,11 \mathrm{~L}$, intervalo de confiança de 95\%: 0,00 a 0,22 L).
Os pacientes tratados com pirfenidona também apresentaram menor risco de diminuição da percentagem prevista de CVF $\geq 10 \%$ ao longo de um ano (odds ratio: 0,58; intervalo de confiança de 95\%: 0,40-0,88), enquanto não houve evidência conclusiva de diferença entre nintedanibe e placebo (OR: 0,65; intervalo de confiança de 95\%: 0,42-1,02).

A NMA indicou que a pirfenidona reduziu a mortalidade por todas as causas em relação ao placebo durante 1 ano (hazard ratio (HR): 0,52, intervalo de confiança de 95\%: 0,28$0,92)$, enquanto para nintedanibe não houve evidência de diferença na mortalidade por todas as causas entre nintedanibe e placebo (HR: 0,70; intervalo de confiança de 95\%: 0,32 a 1,55).

Para a obtenção de ambas as curvas de Kaplan-Meier (KM) utilizadas no modelo, aplicaram-se os HRs obtidos no NMA (Fleetwood et al., 2017) às curvas de sobrevida global e sobrevida livre de progressão dos estudos de pirfenidona (CAPACITY e ASCEND) (King et al., 2014; Noble et al., 2011). Para a definição da extrapolação da curvas de sobrevida global, baseadas nas curvas de KM, uma distribuição paramétrica Weibull foi escolhida (Figura 2). Para a sobrevida livre de progressão, a distribuição paramétrica selecionada foi a lognormal, opção que apresentou o melhor ajuste dos dados (Figura 3).

\section{Descontinuação}

Para cada ciclo, o modelo calculou a proporção de pacientes que continuava ou não no tratamento. Os pacientes que morriam ou recebiam o transplante de pulmão não foram incluídos nos custos de tratamento com pirfenidona ou com nintedanibe. Além disso, os pacientes que recebiam alguma opção terapêutica poderiam descontinuar o tratamento por motivos de eventos adversos, entre outras razões.

A taxa de descontinuação acumulada de pacientes em estados de livre progressão e progressão da doença foi estimada para pirfenidona utilizando o método de KM e os dados do NMA. Como as mortes e os transplantes de pulmão foram obtidos independentemente do modelo, a descontinuação relacionada à morte e ao transplante de pulmão foi censurada na estimativa de KM. Para a extrapolação da curva de descontinuação, a distribuição de Weibull foi a que apresentou melhor ajuste visual aos dados.

\section{Dados de segurança}

Os dados de incidência de eventos adversos foram obtidos pela NMA e estão demonstrados na Tabela 1.

\section{Dados de custos}

Os recursos de saúde considerados se referem aos custos associados ao tratamento medicamentoso, acompanhamento dos pacientes em tratamento, cuidados de fim de vida e o manejo de eventos adversos. 


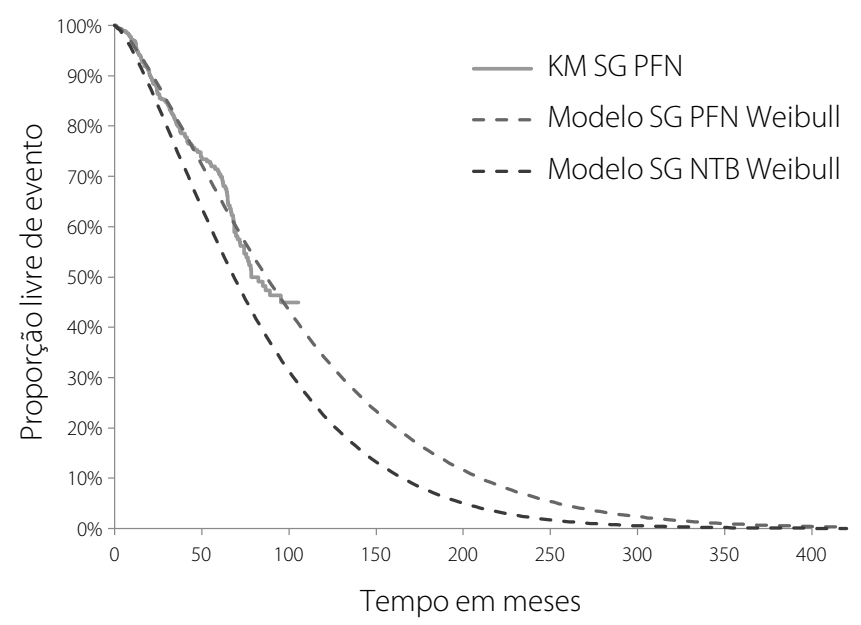

Figura 2. Curvas de sobrevida global.

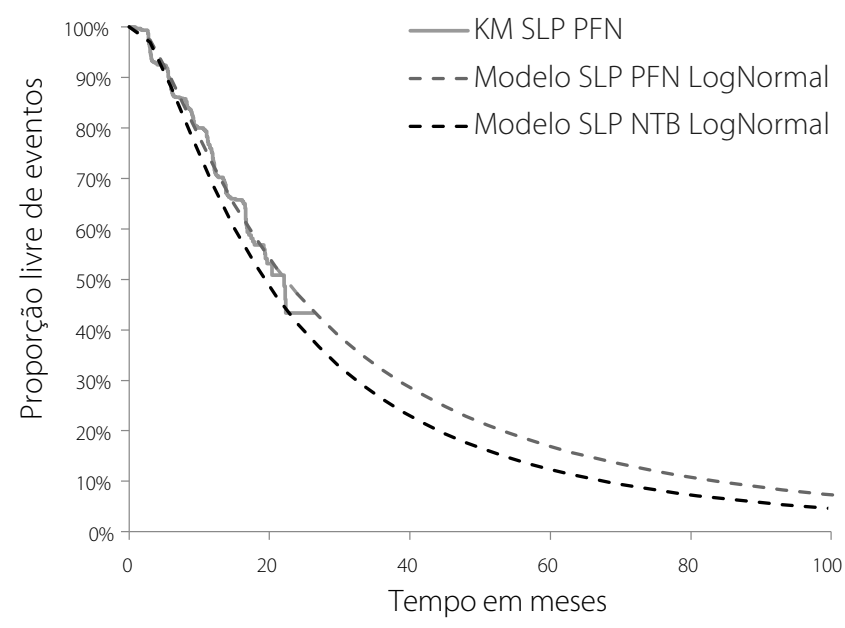

Figura 3. Curvas de sobrevida livre de progressão.

Tabela 1. Incidência de eventos adversos

\begin{tabular}{lcc}
\hline \multicolumn{2}{l}{ Incidência de eventos adversos (por ciclo) } \\
\hline & PFN & NTB \\
\hline Evento cardíaco sério & $0,79 \%$ & $0,23 \%$ \\
\hline Evento gastrointestinal sério & $3,48 \%$ & $1,36 \%$ \\
\hline Perfuração gastrointestinal & - & $0,08 \%$ \\
\hline Reação fotossensitiva & $2,32 \%$ & - \\
\hline
\end{tabular}

NTB: nintedanibe; PFN: pirfenidona.

\section{Tratamento medicamentoso}

O custo unitário da pirfenidona foi obtido a partir da lista de preços da Câmara de Regulação do Mercado de Medicamentos (CMED) (Ministério da Saúde, Brasil. Agência Nacional de Vigilância Sanitária - Anvisa, 2016), com atualização de maio de 2017, considerando-se o preço fábrica com 18\% de ICMS. O custo unitário do medicamento incluído na análise pode ser visualizado na Tabela 2.
Tabela 2. Custo de medicamentos

\begin{tabular}{lcc}
\hline Descrição & $\begin{array}{c}\text { Preço por } \\
\text { frasco-ampola }\end{array}$ & Fonte \\
\hline $\begin{array}{l}\text { Pirfenidona } \\
\text { (267 mg } \times 270 \text { cápsulas) }\end{array}$ & R\$ 9.423,48 & CMED - PF 18\% \\
\hline $\begin{array}{l}\text { Nintedanibe } \\
(150 \text { mg } \times 60 \text { cápsulas) }\end{array}$ & R\$ 15.372,12 & CMED - PF 18\% \\
\hline $\begin{array}{l}\text { CMED: Câmara de Regulamentação do Mercado de Medicamentos; PF: Preço } \\
\text { Fábrica. }\end{array}$
\end{tabular}

\section{Custo de manejo de eventos adversos}

Os custos de manejo de eventos adversos foram baseados em opinião de especialistas e na tabela CBHPM (Classificação Brasileira Hierarquizada de Procedimentos) $5^{\circ}$ edição (AMB Associação Médica Brasileira, 2014) (Tabela 3).

Tabela 3. Custo de tratamento dos eventos adversos

\begin{tabular}{lc}
\hline & Custos \\
\hline Evento cardíaco sério & $R \$ 17.076,98$ \\
\hline Evento gastrointestinal sério & $R \$ 3.961,26$ \\
\hline Perfuração gastrointestinal & $R \$ 17.660,98$ \\
\hline Reação de fotossensibilidade & $R \$ 172,62$ \\
\hline
\end{tabular}

\section{Outros custos}

Os custos referentes aos procedimentos envolvendo transplante de pulmão, custo de fim de vida e acompanhamento do paciente foram baseados em um microcusteio que considerou opinião de especialistas e a lista de procedimentos CBHPM 2015 (AMB, 2014). O custo unitário de transplante de pulmão resultou em $\mathrm{R} \$ 96.681,86$, enquanto o custo de fim de vida foi calculado em $\mathrm{R} \$ 15.524,53$. O custo de manejo de doença foi aplicado em todos os pacientes nos estados de saúde livre de progressão e progressão da doença. Esse custo consistiu nos custos relacionados ao tratamento contínuo e monitoramento da progressão da doença (Tabela 4).

Tabela 4. Custos de acompanhamento da doença

\begin{tabular}{lc}
\hline & Custo \\
\hline Teste de função hepática & $R \$ 72,78$ \\
\hline Transferência de gás & $R \$ 134,66$ \\
\hline Estudo de volume de pulmão & $R \$ 134,66$ \\
\hline Espirometria & $R \$ 154,93$ \\
\hline Teste de exercício & $R \$ 61,96$ \\
\hline Oxigênio & $R \$ 11,00$ \\
\hline $\begin{array}{l}\text { Visita de profissional de } \\
\text { saúde (primeira visita) }\end{array}$ & $R \$ 83,97$ \\
\hline $\begin{array}{l}\text { Visita de professional de saúde } \\
\text { (acompanhamento) }\end{array}$ & $\mathrm{R} \$ 83,97$ \\
\hline Visita do médico & $R \$ 83,97$ \\
\hline
\end{tabular}




\section{Análise de sensibilidade}

Uma análise de sensibilidade foi adaptada para avaliar as principais incertezas do processo e seus respectivos impactos no resultado final. Uma análise de sensibilidade probabilística, em que os parâmetros foram variados de maneira simultânea, foi realizada, admitindo-se mil iterações.

\section{Resultados}

Os resultados comparativos das estratégias alternativas de tratamento foram medidos pela razão de custo-efetividade incremental (RCEI). Esta é definida pelas alternativas de tratamento específicas, como o custo adicional proporcionado pela tecnologia em análise dividido pelo ganho adicional em saúde alcançado por ele.

Os resultados de custo-efetividade demonstraram que a pirfenidona foi dominante quando comparada à opção terapêutica de nintedanibe, como apresentado na Tabela 5.

Por meio da análise de sensibilidade foi possível observar a robustez do modelo, uma vez que $80 \%$ das iterações apresentaram-se no quadrante de menor custo e maior benefício clínico, confirmando o resultado determinístico (Figura 4).

\section{Discussão}

O objetivo do estudo foi avaliar a razão de custo-efetividade incremental entre as opções terapêuticas pirfenidona e nintedanibe no tratamento de pacientes com FPI na perspectiva do sistema suplementar de saúde. Os resultados sugerem que a utilização de pirfenidona é uma opção que tem o po- tencial de promover economia de recursos acompanhado de benefícios clínicos em comparação ao nintedanibe.

Os benefícios clínicos de pirfenidona já foram demonstrados e, em alguns países, esse medicamento já está incluído nas listas de medicamentos recomendados por agências de avaliação de tecnologias em saúde internacionais. Como exemplos relevantes pode-se citar o Canadian Agency for Drugs and Technologies in Health (CADTH), National Institute for Health and Care Excellence (NICE) e Scottish Medicines Consortium, assim como outros países europeus como França, Alemanha, Grécia, Itália, Holanda e Espanha (Canadian Agency for Drugs and Technologies in Health - CADTH, 2015).

Tabela 5. Resultados de custo-efetividade: pirfenidona vs. nintedanibe no horizonte de tempo lifetime

\begin{tabular}{lcccc}
\hline & Pirfenidona & Nintedanibe & $\begin{array}{c}\text { Incremental } \\
\text { (vs. NTB) }\end{array}$ \\
\hline Custos & & & \\
\hline Medicamentos & $R \$ 287.309$ & $R \$ 494.556$ & \\
\hline Manejo da doença & $R \$ 10.871$ & $R \$ 9.347$ & \\
\hline Eventos adversos & $R \$ 8.398$ & $R \$ 5.151$ & \\
\hline Hospitalização & $R \$ 3.640$ & $R \$ 3.162$ & \\
\hline Transplante de pulmão & $R \$ 2.611$ & $R \$ 2.347$ & \\
\hline Fim de vida & $R \$ 6.860$ & $R \$ 8.323$ & \\
\hline Custo total & $R \$ 319.689$ & $R \$ 522.887$ & $-R \$ 203.198$ \\
\hline Efetividade & & \multicolumn{3}{c}{0,810} \\
\hline LY & 6,536 & 5,726 & Dominante \\
\hline RCEl (R\$ / LY) & & \multicolumn{3}{c}{} \\
\hline
\end{tabular}

LY:anos de vida salvos; NTB: nintedanibe; RCEl: razão de custo-efetividade incremental.

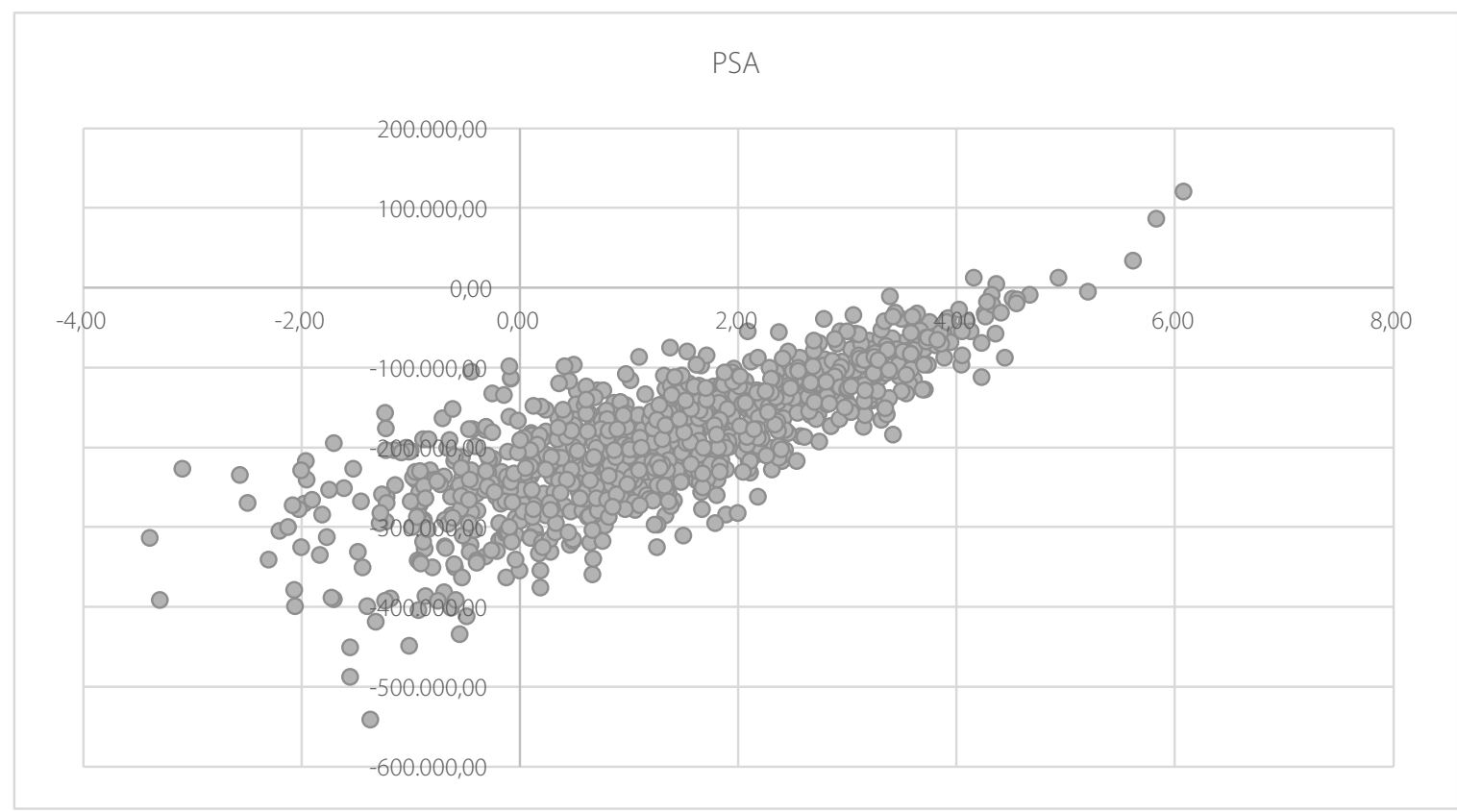

Figura 4. Resultados da análise de sensibilidade probabilística. 
As limitações associadas a essa análise estão relacionadas principalmente às incertezas dos dados clínicos utilizados. Na ausência de estudos de comparação direta entre pirfenidona e nintedanibe, os resultados dessa análise podem não refletir a realidade, uma vez que uma análise de comparação indireta foi utilizada para os cálculos de efetividade. Dessa maneira, os resultados devem ser avaliados com cautela.

Apesar de algumas limitações, o estudo apresenta grande importância devido à ausência de análises econômicas, principalmente de custo-efetividade, de comparação dos tratamentos com pirfenidona e com nintedanibe em pacientes com FPI. A partir desses resultados, é possível ter uma estimativa do possível impacto da adoção de cada um desses tratamentos no sistema de saúde brasileiro.

\section{Conclusão}

A FPI constitui uma doença rara e com elevada carga econômica imposta aos sistemas de saúde. As complicações associadas com a FPI - como hospitalização por exacerbações agudas e infecções do trato respiratório - contribuem para um significativo uso de recursos de saúde, visto que os pacientes muitas vezes necessitam de um longo tempo de permanência hospitalar. Com o progredir da doença, na fase avançada, os pacientes necessitam de terapias onerosas, como, por exemplo, o uso constante de oxigênio, sendo que, em alguns casos, é necessário um grande fornecimento de cilindros de oxigênio que são enviados para as casas dos pacientes (Mooney et al., 2016; Visca et al., 2011).

Nesse sentido e considerando ainda que os sistemas de saúde enfrentam desafios em suas gestões decorrentes do aumento crescente da utilização de recursos e de gastos, a adoção de opções terapêuticas economicamente favoráveis é o mais recomendado no contexto de saúde atual. Dessa forma, são necessárias alternativas terapêuticas que visem à integralidade da assistência farmacêutica de pacientes com FPl e, sobretudo, a sustentabilidade do sistema.

Portanto, considerando esse cenário, verificou-se que a utilização de pirfenidona 267 mg no tratamento de FPI apresenta como uma RCEl dominante em comparação a nintedanibe, ou seja, uma opção terapêutica com menos custos e maior benefício clínico.

\section{Referências bibliográficas}

Adkins J, Collard H. Idiopathic pulmonary fibrosis. Semin Respir Crit Care Med. 2012;33(5):433-9.

AMB - Associação Médica Brasileira. Classificação Brasileira Hierarquizada de Procedimentos Médicos - CBHPM. 2014.

American Thoracic Society (ATS), European Respiratory Society (ERS). American Thoracic Society Idiopathic Pulmonary Fibrosis: diagnosis and treatment - International Consensus Statement. Am J Respir Crit Care Med. 2000;161:646-64.
Bjoraker JA, Ryu JH, Edwin MK, Myers JL, Tazelaar HD, Schroeder DR, et al. Prognostic significance of histopathologic subsets in idiopathic pulmonary fibrosis. Am J Respir Crit Care Med. 1998;157(1):199-203.

Canadian Agency for Drugs and Technologies in Health (CADTH). CDEC Final recommendation Pirfenidone Resubmission (Esbriet - Hoffmann-La Roche Limited). 2015.

Costabel U, Albera C, Bradford WZ, Hormel P, King TE, Noble PW, et al. Analysis of lung function and survival in RECAP: an open-label extension study of pirfenidone in patients with idiopathic pulmonary fibrosis. Sarcoidosis, Vasc Diffus lung Dis. 2014;31(3):198-205.

du Bois RM. An earlier and more confident diagnosis of idiopathic pulmonary fibrosis. Eur Respir Rev. 2012;21(124):141-6.

Fleetwood K, McCool R, Glanville J, Edwards SC, Gsteiger S, Daigl M, et al. Systematic Review and Network Meta-analysis of Idiopathic Pulmonary Fibrosis Treatments. J Manag Care Spec Pharm. 2017;23(Suppl. 3-b):S5-16.

Frankel SK, Schwarz MI. Update in idiopathic pulmonary fibrosis. Curr Opin Pulm Med. 2009;15(5):463-9.

King T, Bradford W, Castro-Bernadini S, Fagan E. The ASCEND Study: A Randomized, Double-Blind, Placebo Controlled Trial of Pirfenidone in Patients with Idiopathic Pulmonary Fibrosis (IPF). ATS 2014 International Conference. 2014.

Kistler KD, Nalysnyk L, Rotella P, Esser D. Lung transplantation in idiopathic pulmonary fibrosis: a systematic review of the literature. BMC Pulm Med. 2014;14:139.

Lee AS, Mira-Avendano I, Ryu JH, Daniels CE. The burden of idiopathic pulmonary fibrosis: An unmet public health need. Respir Med. 2014;108(7):955-67.

Ley B, Collard HR, King TE. Clinical course and prediction of survival in idiopathic pulmonary fibrosis. Am J Respir Crit Care Med. 2011;183(4):431-40.

Medscape. Idiopathic Pulmonary Fibrosis [Internet]. 2016. Disponível em: goo.gl/KiXMTP. Acesso em: maio 2017.

Meltzer EB, Noble PW. Idiopathic pulmonary fibrosis. Orphanet J Rare Dis. 2008;3:8.

Ministério da Saúde (Brasil). Agência Nacional de Vigilância Sanitária (Anvisa). Câmara de Regulação de Mercado de Medicamentos [Internet]. 2016. Disponível em: http://portal.anvisa.gov.br/cmed. Acesso em: maio 2017.

Ministério da Saúde (Brasil). Secretária de Ciência, Tecnologia e Insumos Estratégicos. Departamento de Ciência e Tecnologia. Diretrizes metodológicas: elaboração de pareceres técnico-científicos. 4 ed. Brasília: Ministério da Saúde; 2014.80 p.

Mooney J, Raimundo K, Chang E, Broder M. Mortality, costs, and length of stay in patients with idiopathic pulmonary fibrosis. Predictors of outcome in ILD. 2016. p. A4286.

Nalysnyk L, Cid-Ruzafa J, Rotella P, Esser D. Incidence and prevalence of idiopathic pulmonary fibrosis: review of the literature. Eur Respir Rev. 2012;21(126):355-61.

Nathan SD, Brown AW, King CS. Guide to Clinical Management of Idiopathic Pulmonary Fibrosis [Internet]. Cham: Springer International Publishing; 2016. Disponível em: http://link.springer.com/10.1007/978-3-319-32794-5.

Noble PW, Albera C, Bradford WZ, Costabel U, Glassberg MK, Kardatzke $D$, et al. Pirfenidone in patients with idiopathic pulmonary fibrosis (CAPACITY): two randomised trials. Lancet. 2011;377(9779):1760-9.

Nunes H, Carton Z, Cottin V, Israel-Biet D, Brauner M, Kambouchner M, et al. Preliminary results of the French national prospective cohort on IPF. European Respiratory J. 2011;38(Suppl. 55):P646.

Raghu G, Collard HR, Egan JJ, Martinez FJ, Behr J, Brown KK, et al. An Official ATS/ERS/JRS/ALAT Statement: Idiopathic pulmonary fibrosis: Evidencebased guidelines for diagnosis and management. Am J Respir Crit Care Med. 2011;183(6):788-824. 
Raghu G, Rochwerg B, Zhang Y, Garcia CAC, Azuma A, Behr J, et al. An official ATS/ERS/JRS/ALAT clinical practice guideline: Treatment of idiopathic pulmonary fibrosis: an update of the 2011 clinical practice guideline. Am J Respir Crit Care Med. 2015;192(2):e3-19.

Richeldi L, Costabel U, Selman M, Kim D, Hansell D, Nicholson A, et al. Efficacy of a Tyrosine Kinase Inhibitor in Idiopathic Pulmonary Fibrosis. N Eng J Med. 2011;365(12):1079-87.
Stowasser S, Hallmann C, Lancet T, Raghu G, Rochwerg B, Zhang Y, et al. New guideline for idiopathic pulmonary fibrosis. Lancet. 2015;386(10006):1823-4.

Visca D, Montgomery A, de Lauretis A, Sestini P, Soteriou H, Maher TM, et al. Ambulatory oxygen in interstitial lung disease. Eur Respir J. 2011;38(4):987-90. 